\title{
Comparative Analysis of Drought Indices for Drought Zone Scheme of Northern Khorasan Province of Iran
}

\author{
Ehsan EYSHI REZAEI ${ }^{1 *}$, Azade MOHAMMADIAN² \\ Mansoreh KOOHI², Mohammad BANNAYAN' \\ ${ }^{1}$ Ferdowsi University of Mashhad, Faculty of Agriculture, P.O. Box, 91775-1163, Mashhad, \\ Iran;ch_ey145@stu-mail.um.ac.ir (*orresponding author) \\ ${ }^{2}$ Climatological Research Institute, Climatology of Atmospheric Disasters Group, P. O. Box: 91735-676, Mashhad, Iran
}

\begin{abstract}
Drought is one of the natural disasters which deeply influenced agricultural production. Drought monitoring programs could help to forecast and mitigate the impacts of drought. In this study occurrence, severity, and duration of drought were evaluated by monthly rainfall data (1986-2005) that were recorded at all meteorological stations in north Khorasan province of Iran. Drought indices include Standard Rainfall Index (SPI), Decades Index (DI) and Percent of Normal (PNI) calculated and compared to determine drought severity, duration and drought occurrence for all stations. In addition, drought maps were prepared by Inverse Distance Weighted (IDW) method, for each study zone. Based on these indices, the most extensive drought occurred in 1989, 1990, 1995, 1996, 2000, and 2001 years. The longest duration of drought based on SPI happened in 1994 and 1997 years. Furthermore, the extreme drought occurred in 1990 and 2001 in all stations. In conclusion, Central part of this province was more exposed to extreme drought during study period than other parts of this region.
\end{abstract}

Keywords: drought, geostatistic, indices, Khorasan, rainfall

\section{Introduction}

Human life across the history of planet was always exposed to lots of natural hazards. Some of these hazards caused by geological activities like earthquakes or volcanoes, and some by natural phenomena such as drought which driving by climate. Drought is one of the major natural hazards around the world, and it occurs almost every year across the world. Prediction plans are necessary to prepare for mitigating the drought affects for putting behind this event with minimum economic and social losses. On the other hand, management of drought is necessary by existence of comprehensive forecast systems. Drought indices are great tools for drought forecast systems (Gourabi, 2004). A large number of drought indices exist and each requires variety of data input and provides somewhat different measure of drought (Kao and Govindaraju, 2010).

Standard Rainfall Index (SPI) developed by McKee et al. (1993), to quantify the rainfall deficit effects on groundwater, surface supplies of water, soil moisture and flow channels on Colorado State of America. This index has been used as a deficit measure in many studies and could show rainfall deficit effects on water supplies. Rainfall deficit usually in short period affects the soil moisture but over long period affects the groundwater and rivers flows (Guttman, 1998). McKee et al. (1993), used SPI on deferent time scales $(3,6,12,24,48$ months) and the rainfall records were fit to a probability distribution function and subsequently transformed to standard normal distribution function (Guttman, 1998). This insured that the mean SPI value for any given location (and duration) would be zero and the variance would be one.

Decades Index (DI) introduced by Gibbs and Maher (1967), according to this method rainfall distribution in a long period should be divided to ten parts. First part refers to the amount of rainfall that would be less than ten percentages of overall rainfall and continues arranged until tenth part. Rainfall is the heart of this index calculation and time scale for measurement of it could be month, season or year.

Percent of Normal (PNI) index identified by Willeke et al. (1998) as a measure of observed drought. Main concept of this index was based on the ratio of the real rainfall to normal rainfall.

Percent of Normal (PNI) index used by long-term average of rainfall for base values and then rainfall changes during monthly, seasonally and annual scales. Akhtari et al. (2007) investigated drought occurrence using SPI and Effective Drought Index (EDI) indices for Tehran province of Iran and employed geostatistic methods to illustrate maps of the spatial distribution of drought. Ansari (2008) studied drought intensity by SPI index in Central khorasan region, and classified Central Khorasan as dry area. Rahemi (2005) evaluated drought periods for Azer- 
63

baijan region by SPI index and determined dry years for this region.

Utilization of geostatistic methods was a common way to estimate the climatic condition of different locations where there was some data gaps (Goovaerts, 2000). Geostatistical methods have been used for required extrapolation to somewhere which climate station did not exist (Akhtari et al., 2007). Ansari (2008) reported that Kriging and IDW (Inverse Distance Weighted) methods showed highest performance across different methods of extrapolation for drought zone scheme. Inverse distance weighted method is based on the assumption that the interpolating surface should be influenced mostly by the nearby points and less by the more distant points. The interpolating surface is a weighted average of the scatter points and the weight assigned to each scatter point diminishes as the distance from the interpolation point to the scatter point increases (Abtew et al., 1993).

The main aims of this study were: the determination of drought occurrence periods and intensities across the northern Khorasan province of Iran by different drought indices (1), the comparison of different drought indices (2) and the manufacturing a zone scheme of northern Khorasan province from drought perspective with Inverse Distance Weighted method (3).

\section{Materials and methods}

Northern Khorasan province is on $55^{\circ} 58^{\prime} \mathrm{N}$ latitude to $36^{\circ} 38^{\prime}$ W longitude and covered about $28100 \mathrm{Km}^{2}$ of Iran's area (Fig. 1). Monthly rainfall was the only available long-term data for northern Khorasan province across 17 meteorological stations for drought indices calculation. All data have been initially tested to be normalized. In this study, three standardized metrological indices were used; include Standard Rainfall Index (SPI), Decades Index (DI) and Percent of Normal Index (PNI).

The SPI is based on the cumulative probability of a given rainfall event at a station (McKee et al., 1993). SPI was calculated as:

$$
S P I=\frac{X-\bar{X}}{\delta}
$$

Where $X$ is the rainfall quantities (monthly or annually), $\bar{X}$ is the average rainfall of study period and $\delta$ is standard deviation of rainfall data. The SPI could effectively represent the amount of rainfall over a given time scale, with the advantage to provide not only information on the amount of rainfall, but that it also gives an indication of what this amount is in relation to the normality. Quantities and descriptive situations of this index are shown in Tab. 1. SPI was calculated by SPI-SL-6 software (Rossi et al., 2007).

Decades Index is calculated based on rainfall distribution of long term data, and were divided to ten parts. First part referrals amount of rainfall that less than ten percent-

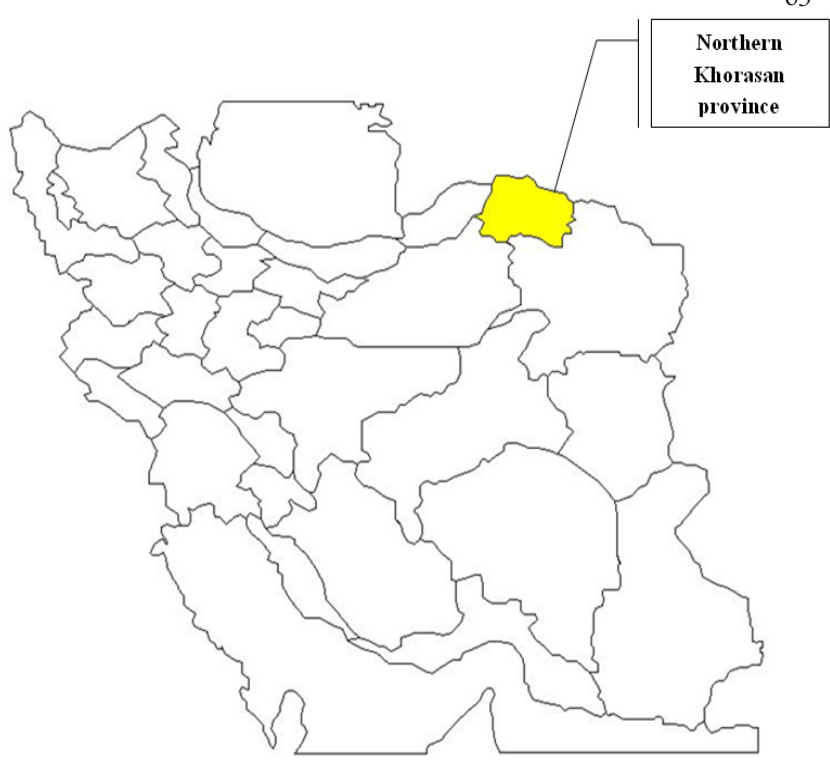

Fig. 1. Northern Khorasan province location in Iran

ages of overall rainfall and continues arranged until tenth part (Tab. 1). Categorization of Decades was performed by SPSS software (Leech et al., 2005).

Percent of Normal Index also calculated as:

$$
\% p n=(p i / n p) \times 100
$$

Where $\% p n$ is the percent of normal rainfall, $p i$ is the rainfall in $i$ year, and $n p$ is average of rainfall of study period. Descriptive features of values related to this index shown in Tab. 1. For this study, it assumed that if drought occurred at all levels (extreme, severe, and slight) based on the three testing methods, at nine or more stations for a year, then drought situation would be considered across the whole province for that specific year. Inverse Distance Weighted (IDW) method was also used for geographical drought zone scheme and mapping (Breslin et al., 1999).

\section{Results and discussion}

\section{Drought duration and intensity}

Based on our assumption criteria, which was defined in methods section, drought occurred across northern Khorasan province in six years $(1989,1990,1995,1996$, 2000, and 2001) during study period (1986-2005), but drought intensity was different at different stations. Rainfall values in all stations, during drought occurrence years represented in Fig. 2. Quantification of drought for above six years using SPI is presented in Tab. 2. SPI has considerable capability for calculation drought occurrence for each month of the year as this index is able to show drought onset, duration, and cessation. Five-drought periods were determined for this study period (Tab. 3). According to SPI test, the longest period of drought occurred in 1994 to 1997 , therefore, the highest damage to rainfed crops was resulted in those years. Extreme drought occurred in 1990 and 2001 years especially in 1990 that Iran and Mid- 
64

Tab. 1. Classifications of Decades Index (DI), Standard Precipitation Index (SPI) and Percent of Normal Index (PNI)

\begin{tabular}{|c|c|c|c|c|c|c|}
\hline Decades & Situations & Percentage of occurrence & Situations & Values of SPI & Situations & Values of PNI \\
\hline 1 & Extreme drought & Less than 10 percent & Extreme drought & $\mathrm{SPI} \leq-2$ & Extreme drought & $<40$ \\
\hline 2 & Severe drought & 10 to 20 percent & Severe drought & $-1.5 \leq \mathrm{SPI} \leq-1.99$ & Severe drought & $40-55$ \\
\hline 3 & Moderate drought & 20 to 30 percent & Moderate drought & $-1 \leq \mathrm{SPI} \leq-1.4$ & Moderate drought & $55-70$ \\
\hline 4 & Slight drought & 30 to 40 percent & Normal & $-0.99 \leq \mathrm{SPI} \leq 0.99$ & Slight drought & $70-80$ \\
\hline 5 & Normal & 40 to 50 percent & Mediate humidity & $1 \leq \mathrm{SPI} \leq 1.49$ & Normal & $>80$ \\
\hline 6 & Mild humidity & 50 to 60 percent & - & - & - & - \\
\hline
\end{tabular}

Tab. 2. Values and situations of drought occurrence across Northern Khorasan province by Standard Precipitation Index

\begin{tabular}{|cccccccccccccc}
\hline Station & 1989 & Situation & 1990 & Situation & 1995 & Situation & 1996 & Situation & 2000 & Situation & 2001 & Situation \\
\hline Bojnurd & -0.34 & $\mathrm{~N}$ & -2.25 & $\mathrm{ED}$ & -1.08 & $\mathrm{MD}$ & -1.11 & $\mathrm{MD}$ & 0.59 & $\mathrm{~N}$ & -2.16 & $\mathrm{ED}$ \\
\hline Seasab & -0.93 & $\mathrm{~N}$ & -2.06 & $\mathrm{ED}$ & -1.37 & $\mathrm{MD}$ & -1.2 & $\mathrm{MD}$ & -1.15 & $\mathrm{MD}$ & -1.01 & $\mathrm{MD}$ \\
\hline Ashraf Darreh & -0.74 & $\mathrm{~N}$ & -1.88 & $\mathrm{SD}$ & -0.77 & $\mathrm{~N}$ & -0.07 & $\mathrm{~N}$ & -0.13 & $\mathrm{~N}$ & -1.35 & $\mathrm{MD}$ \\
\hline Balaghan & -1.98 & $\mathrm{SD}$ & -1.4 & $\mathrm{MD}$ & -1.61 & $\mathrm{SD}$ & -0.23 & $\mathrm{~N}$ & -1.16 & $\mathrm{MD}$ & -1.85 & $\mathrm{SD}$ \\
\hline Fajr Abad & -1 & $\mathrm{MD}$ & -2.13 & $\mathrm{ED}$ & -0.97 & $\mathrm{~N}$ & -0.06 & $\mathrm{~N}$ & -0.39 & $\mathrm{~N}$ & -0.9 & $\mathrm{~N}$ \\
\hline Farooj & -1.86 & $\mathrm{SD}$ & -1.9 & $\mathrm{SD}$ & -0.62 & $\mathrm{~N}$ & -0.68 & $\mathrm{~N}$ & -0.68 & $\mathrm{~N}$ & -1.34 & $\mathrm{MD}$ \\
\hline Golian & -1.41 & $\mathrm{SD}$ & -1.3 & $\mathrm{MD}$ & -1.38 & $\mathrm{MD}$ & -0.22 & $\mathrm{~N}$ & -2.17 & $\mathrm{ED}$ & -1.11 & $\mathrm{MD}$ \\
\hline Jajarm & -0.26 & $\mathrm{~N}$ & -1.48 & $\mathrm{MD}$ & -0.83 & $\mathrm{~N}$ & 0.72 & $\mathrm{~N}$ & -0.99 & $\mathrm{~N}$ & -1.76 & $\mathrm{SD}$ \\
\hline Kalateh Bahar & -0.91 & $\mathrm{~N}$ & -2.42 & $\mathrm{ED}$ & -0.61 & $\mathrm{~N}$ & -0.26 & $\mathrm{~N}$ & -0.45 & $\mathrm{~N}$ & -1.38 & $\mathrm{MD}$ \\
\hline Langar & -0.19 & $\mathrm{~N}$ & -2.6 & $\mathrm{ED}$ & -1.11 & $\mathrm{MD}$ & -0.12 & $\mathrm{~N}$ & 0.05 & $\mathrm{~N}$ & -1.28 & $\mathrm{MD}$ \\
\hline Mangoli & -0.67 & $\mathrm{~N}$ & -1.31 & $\mathrm{MD}$ & -1.76 & $\mathrm{SD}$ & 0.07 & $\mathrm{~N}$ & -1.38 & $\mathrm{MD}$ & -0.35 & $\mathrm{~N}$ \\
\hline Mokhaberat & -1.06 & $\mathrm{MD}$ & -2.78 & $\mathrm{ED}$ & 0.02 & $\mathrm{~N}$ & 0.01 & $\mathrm{~N}$ & 0.14 & $\mathrm{~N}$ & -1.19 & $\mathrm{MD}$ \\
\hline Sarcheshmeh & -1.31 & $\mathrm{MD}$ & -1.09 & $\mathrm{MD}$ & -1.01 & $\mathrm{MD}$ & -0.22 & $\mathrm{~N}$ & -1.6 & $\mathrm{MD}$ & -2.1 & $\mathrm{ED}$ \\
\hline Sardab & -0.18 & $\mathrm{~N}$ & -1.29 & $\mathrm{MD}$ & -1.61 & $\mathrm{SD}$ & -0.57 & $\mathrm{~N}$ & -1.58 & $\mathrm{SD}$ & -1.57 & $\mathrm{SD}$ \\
\hline Takleh Ghooz & -0.31 & $\mathrm{~N}$ & -1.72 & $\mathrm{SD}$ & -1.6 & $\mathrm{SD}$ & -1.48 & $\mathrm{MD}$ & 0.25 & $\mathrm{~N}$ & -1.51 & $\mathrm{MD}$ \\
\hline Tazeh Ghaleh & -0.5 & $\mathrm{~N}$ & -1.83 & $\mathrm{SD}$ & -1.19 & $\mathrm{MD}$ & -0.69 & $\mathrm{~N}$ & -0.5 & $\mathrm{~N}$ & -0.96 & $\mathrm{~N}$ \\
\hline Yengi Ghaleh & -1.11 & $\mathrm{MD}$ & -1.5 & $\mathrm{SD}$ & -1.14 & $\mathrm{MD}$ & -0.9 & $\mathrm{~N}$ & -1.09 & $\mathrm{MD}$ & -1.79 & $\mathrm{SD}$ \\
\hline
\end{tabular}

ED: Extreme drought, SD: Severe drought, MD: Moderate drought, and N: Normal

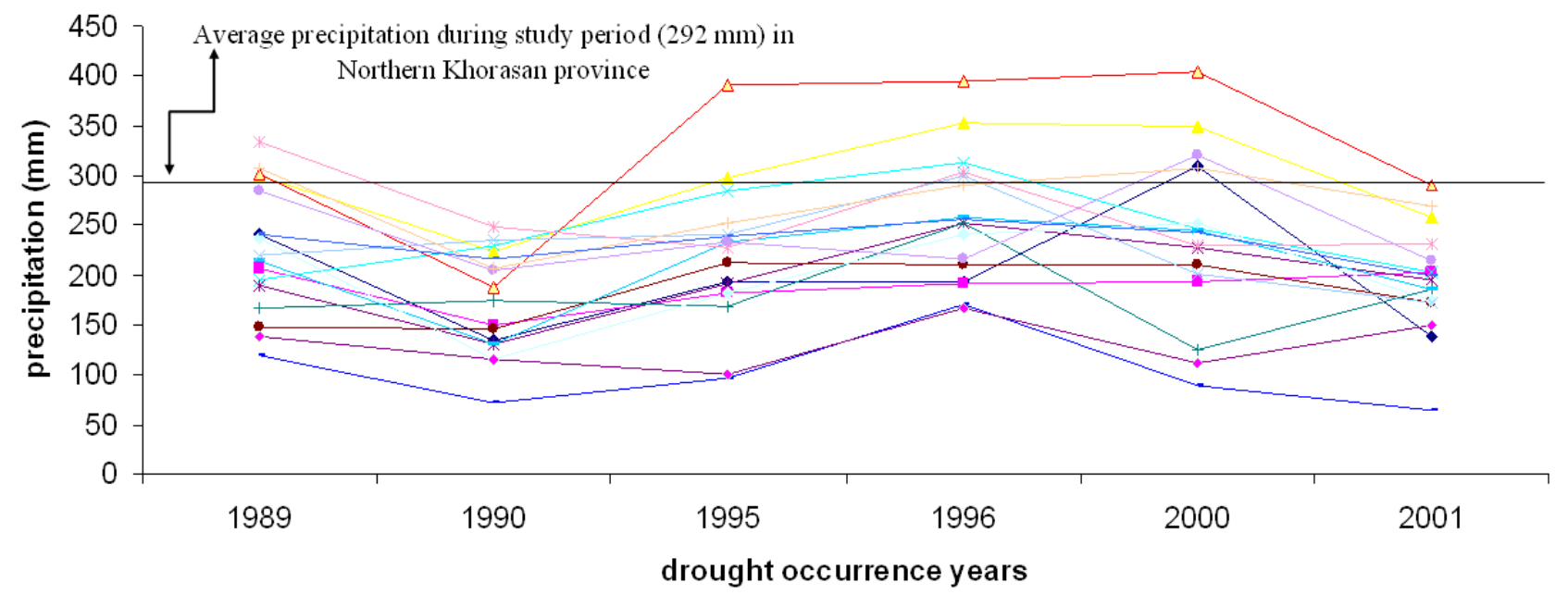

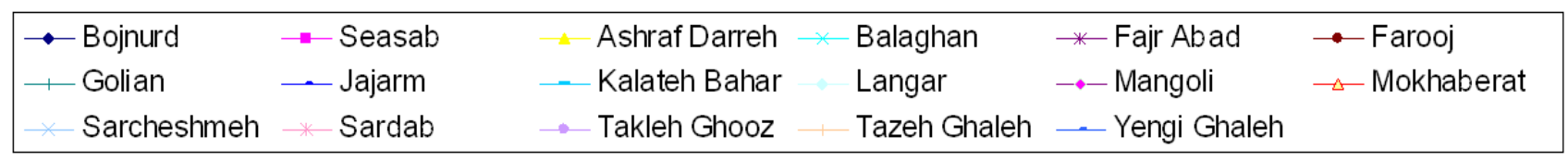

Fig. 2. Precipitation values during drought occurrence years in Northern Khorasan province 
Tab. 3. Drought occurrence periods for 12 months time sires by Standard Precipitation Index during study period (1989-2005) for all stations

\begin{tabular}{|c|c|c|c|c|c|c|c|c|c|c|c|c|c|c|c|c|c|c|c|c|}
\hline \multirow{2}{*}{ Station } & \multicolumn{4}{|c|}{ First drought period } & \multicolumn{4}{|c|}{ Second drought period } & \multicolumn{4}{|c|}{ Third drought period } & \multicolumn{4}{|c|}{ Forth drought period } & \multicolumn{4}{|c|}{ Fifth drought period } \\
\hline & $S$ & $E$ & $\mathrm{C}$ & $\mathrm{I}$ & $S$ & $E$ & $\mathrm{C}$ & $\mathrm{I}$ & $S$ & $\mathrm{E}$ & $\mathrm{C}$ & $\mathrm{I}$ & $S$ & $E$ & $\mathrm{C}$ & $\mathrm{I}$ & $S$ & $\mathrm{E}$ & $\mathrm{C}$ & $\mathrm{I}$ \\
\hline Bojnurd & 1989.7 & 1991.4 & 22 & -2.25 & 1994.4 & 1997.9 & 42 & -1.87 & 1998.11 & 1999.9 & 11 & -1.55 & 2000.5 & 2000.8 & 4 & -1.13 & 2001.9 & 2002.3 & 7 & -2.3 \\
\hline Seasab & 1989.4 & 1991.4 & 23 & -2.06 & 1994.6 & 1997.5 & 36 & -1.77 & 2000.2 & 2002.2 & 25 & -2.71 & - & - & - & - & - & - & - & - \\
\hline Ashraf Darreh & 1989.5 & 1991.2 & 35 & -2.48 & 1995.1 & 1996.1 & 13 & -1.09 & 1997.3 & 1997.4 & 1 & -1.22 & 1993.3 & 2000.8 & 18 & -1.8 & 2001.9 & 2002.1 & 5 & -1.61 \\
\hline Balaghan & 1989.3 & 1992.3 & 26 & -2.32 & 1994.5 & 1995.3 & 11 & -0.92 & 1995.12 & 1996.4 & 5 & -0.68 & 1997.2 & 1997.5 & 4 & -1 & 2000.3 & 2002.3 & 25 & -2.17 \\
\hline Fajr Abad & 1989.4 & 1991.4 & 32 & -2.24 & 1994.11 & 1996.1 & 15 & -1.89 & 1997.2 & 1997.6 & 5 & -1.54 & 2000.5 & 2002.8 & 21 & -1.32 & 2004.5 & 2005.12 & 20 & -1.15 \\
\hline Farooj & 1989.4 & 1991.11 & 32 & -2.16 & 1994.4 & 1995.1 & 10 & -1.45 & 1995.12 & 1997.5 & 18 & -1.29 & 2000.4 & 2002.3 & 24 & -1.67 & - & - & - & - \\
\hline Golian & 1989.3 & 1991.3 & 25 & -1.98 & 1993.7 & 1993.10 & 4 & -0.64 & 1995.5 & 1997.5 & 25 & -1.56 & 2000.3 & 2002.3 & 23 & -2.27 & 2004.4 & 2004.12 & 9 & -1.56 \\
\hline Jajarm & 1989.4 & 1991.2 & 23 & -1.48 & 1994.4 & 1994.9 & 6 & -0.55 & 1995.12 & 1996.2 & 3 & -0.88 & 1999.8 & 2002.3 & 39 & -2.02 & 2004.4 & 2004.12 & 9 & -0.91 \\
\hline Kalateh Bahar & 1989.6 & 1991.12 & 31 & -2.82 & 1994.5 & 1996.1 & 21 & -1.23 & 1997.3 & 1997.4 & 2 & -0.97 & 1999.5 & 2002.3 & 35 & -1.8 & - & - & - & - \\
\hline Langar & 1989.8 & 1991.4 & 21 & -2.6 & 1994.4 & 1996.10 & 31 & -1.62 & 1997.4 & 1997.9 & 6 & -0.88 & 1998.12 & 1999.12 & 13 & -1.9 & 2001.1 & 2002.3 & 15 & -1.79 \\
\hline Mangoli & 1989.9 & 1991.2 & 18 & -1.53 & 1993.6 & 1994.10 & 17 & -1.55 & 1995.11 & 1996.10 & 12 & -1.82 & 1997.4 & 1997.12 & 9 & -1.15 & 2000.3 & 2001.11 & 21 & -2.28 \\
\hline Mokhaberat & 1987.8 & 1988.5 & 10 & -1.27 & 1989.8 & 1991.4 & 21 & -2.83 & 1993.5 & 1993.12 & 8 & -1.29 & 1994.4 & 1995.5 & 14 & -1.6 & 2001.9 & 2002.3 & 7 & -1.52 \\
\hline Sarcheshmeh & 1989.5 & 1991.3 & 23 & -2.02 & 1995.11 & 1997.10 & 24 & -1.38 & 2000.2 & 2002.3 & 26 & -2.53 & 2003.12 & 2004.10 & 11 & -1.58 & - & - & - & - \\
\hline Sardab & 1989.8 & 1991.4 & 21 & -1.3 & 1994.12 & 1996.3 & 16 & -1.73 & 1996.12 & 1997.5 & 6 & -1.84 & 1998.12 & 1999.10 & 11 & -1.62 & 2000.3 & 2002.3 & 25 & -2.06 \\
\hline Takleh Ghooz & 1987.6 & 1987.9 & 4 & -1.22 & 1989.5 & 1991.11 & 31 & -1.73 & 1995.11 & 1997.4 & 18 & -1.78 & 1999.3 & 2000.7 & 17 & -2.59 & 2001.9 & 2002.2 & 6 & -1.51 \\
\hline Tazeh Ghaleh & 1989.12 & 1987.11 & 12 & -1.59 & 1989.5 & 1991.12 & 32 & -1.83 & 1995.1 & 1997.4 & 28 & -1.48 & 1999.5 & 1999.10 & 6 & -1.18 & 2000.11 & 2001.12 & 14 & -1.35 \\
\hline Yengi Ghaleh & 1989.4 & 1991.4 & 25 & -1.61 & 1995.1 & 1997.4 & 28 & -1.4 & 1998.1 & 1999.10 & 13 & -1.1 & 2000.4 & 2002.2 & 23 & -2.07 & 2004.4 & 2004.9 & 6 & -1.16 \\
\hline
\end{tabular}

S: Start (year/month), E: End (year/month), C: Continuity (month) and I: Intensity. 
66

Tab. 4. Values and situations of drought occurrence across Northern Khorasan province by Decades Index

\begin{tabular}{cccccccccccccc}
\hline Station & 1989 & Situation & 1990 & Situation & 1995 & Situation & 1996 & Situation & 2000 & Situation & 2001 & Stuation \\
\hline Bojnurd & 3 & MED & 1 & ED & 2 & SD & 2 & SD & 7 & MH & 1 & ED \\
\hline Seasab & 3 & MOD & 1 & ED & 1 & ED & 2 & SD & 2 & SD & 3 & MED \\
Ashraf Darreh & 3 & MOD & 1 & ED & 3 & MOD & 5 & N & 4 & MD & 2 & SD \\
\hline Balaghan & 1 & TSD & 2 & SD & 3 & MOD & 4 & S & 2 & SD & 1 & ED \\
\hline Fajr Abad & 2 & SD & 1 & ED & 2 & SD & 6 & N & 5 & N & 3 & MED \\
\hline Farooj & 1 & ED & 1 & ED & 4 & S & 3 & MOD & 3 & MOD & 2 & SD \\
Golian & 1 & ED & 2 & SD & 2 & SD & 4 & S & 1 & ED & 3 & MED \\
\hline Jajarm & 4 & MD & 1 & ED & 3 & S & 9 & HH & 2 & SD & 1 & ED \\
Kalateh Bahar & 2 & SD & 1 & ED & 3 & MOD & 5 & N & 4 & MD & 1 & ED \\
\hline Langar & 5 & N & 1 & ED & 2 & SD & 5 & N & 6 & N & 1 & ED \\
Mangoli & 3 & MOD & 2 & SD & 1 & ED & 5 & N & 2 & SD & 4 & MD \\
\hline Mokhaberat & 2 & SD & 1 & ED & 4 & S & 4 & S & 6 & N & 2 & SD \\
Sarcheshmeh & 2 & SD & 2 & SD & 3 & MOD & 4 & S & 1 & ED & 1 & ED \\
Sardab & 5 & N & 2 & SD & 1 & ED & 4 & S & 1 & ED & 2 & SD \\
\hline Takleh Ghooz & 3 & MOD & 1 & ED & 2 & SD & 2 & SD & 6 & N & 1 & ED \\
\hline Tazeh Ghaleh & 4 & MD & 1 & ED & 2 & SD & 3 & MOD & 4 & S & 2 & SD \\
Yengi Ghaleh & 2 & SD & 1 & ED & 2 & SD & 3 & MOD & 3 & MOD & 1 & ED \\
\hline
\end{tabular}

ED: Extreme drought, SD: Severe drought, MOD: Moderate drought, S: Slight drought, N: Normal, and MH: Mild humidity

dle East regions exposed with severe drought (Rahemi, 2005).

Decades Index could show only drought intensity in specific time scales (Wilhite, 2005), thus it is not possible to calculate drought duration by Decades Index. Annually drought intensities in drought occurrence years throughout northern Khorasan province by Decades Index approach were shown in Tab. 4. According to Decades Index testing method, severe drought occurrences were in 1990 and 2001 years.
Percent of Normal Index is one of the simplest and applicable methods to investigate about drought occurrence but rainfall data with normal distribution is necessary for accurate determination of drought intensity with this method. Rainfall data in this study followed the normal distribution. This index like Decades Index only showed drought intensity. According to Percent of Normal Index the most drought intensities were in 1990 and 2001 years (Tab. 5).

Droughts intensities showed different situations during drought occurrence years across all stations which

Tab. 5. Values and situations of drought occurrence across Northern Khorasan province by Percent of Normal Index

\begin{tabular}{|c|c|c|c|c|c|c|c|c|c|c|c|c|}
\hline Station & 1989 & Situation & 1990 & Situation & 1995 & Situation & 1996 & Situation & 2000 & Situation & 2001 & Situation \\
\hline Bojnurd & 88.8 & $\mathrm{~N}$ & 49.6 & SD & 71.8 & $S$ & 71.2 & $S$ & 114.1 & $\mathrm{~N}$ & 51.1 & SD \\
\hline Seasab & 76.4 & S & 55.4 & MOD & 67.5 & MOD & 70.9 & $S$ & 71.9 & MOD & 74.8 & MOD \\
\hline Ashraf Darreh & 82.1 & $\mathrm{~N}$ & 61.1 & MOD & 81.5 & $\mathrm{~N}$ & 96.6 & $\mathrm{~N}$ & 95.2 & $\mathrm{~N}$ & 70.2 & MOD \\
\hline Balaghan & 57.7 & MOD & 67.9 & MOD & 84.0 & $\mathrm{~N}$ & 92.5 & $\mathrm{~N}$ & 72.6 & MOD & 59.9 & MOD \\
\hline Fajr Abad & 72.6 & $S$ & 50.0 & SD & 73.1 & S & 95.9 & $\mathrm{~N}$ & 87.0 & $\mathrm{~N}$ & 74.7 & MOD \\
\hline Farooj & 57.2 & MOD & 56.6 & MOD & 82.6 & $\mathrm{~N}$ & 81.2 & $\mathrm{~N}$ & 81.2 & $\mathrm{~N}$ & 67.2 & MOD \\
\hline Golian & 59.9 & MOD & 62.4 & MOD & 60.5 & MOD & 90.1 & $\mathrm{~N}$ & 44.7 & SD & 66.8 & MOD \\
\hline Jajarm & 86.6 & $\mathrm{~N}$ & 52.2 & SD & 69.1 & MOD & 123.7 & $\mathrm{~N}$ & 64.7 & MOD & 45.9 & SD \\
\hline Kalateh Bahar & 75.0 & S & 46.2 & SD & 82.0 & $\mathrm{~N}$ & 90.7 & $\mathrm{~N}$ & 85.8 & $\mathrm{~N}$ & 65.1 & MOD \\
\hline Langar & 97.6 & $\mathrm{~N}$ & 47.6 & SD & 75.5 & $S$ & 99.4 & $\mathrm{~N}$ & 103.8 & $\mathrm{~N}$ & 71.8 & MOD \\
\hline Mangoli & 81.6 & $\mathrm{~N}$ & 68.1 & MOD & 59.6 & MOD & 99.5 & $\mathrm{~N}$ & 66.7 & MOD & 89.1 & $\mathrm{~N}$ \\
\hline Mokhaberat & 75.9 & S & 47.4 & SD & 98.7 & $\mathrm{~N}$ & 99.2 & $\mathrm{~N}$ & 101.6 & $\mathrm{~N}$ & 73.2 & MOD \\
\hline Sarcheshmeh & 69.2 & MOD & 73.9 & $S$ & 75.7 & $S$ & 81.9 & $\mathrm{~N}$ & 58.3 & MOD & 45.7 & SD \\
\hline Sardab & 91.7 & $\mathrm{~N}$ & 68.4 & MOD & 62.5 & MOD & 83.1 & $\mathrm{~N}$ & 62.0 & MOD & 63.3 & MOD \\
\hline Takleh Ghooz & 91.9 & $\mathrm{~N}$ & 66.1 & MOD & 75.8 & $S$ & 70.2 & S & 104.0 & $\mathrm{~N}$ & 69.5 & MOD \\
\hline Tazeh Ghaleh & 85.3 & $\mathrm{~N}$ & 57.6 & MOD & 70.1 & S & 80.9 & $\mathrm{~N}$ & 85.3 & $\mathrm{~N}$ & 74.8 & MOD \\
\hline Yengi Ghaleh & 74.1 & $S$ & 66.7 & MOD & 73.6 & $S$ & 78.4 & $S$ & 75.1 & MD & 57.7 & MOD \\
\hline
\end{tabular}

ED: Extreme drought, SD: Severe drought, MOD: Moderate drought, S: Slight drought, and N: Normal 

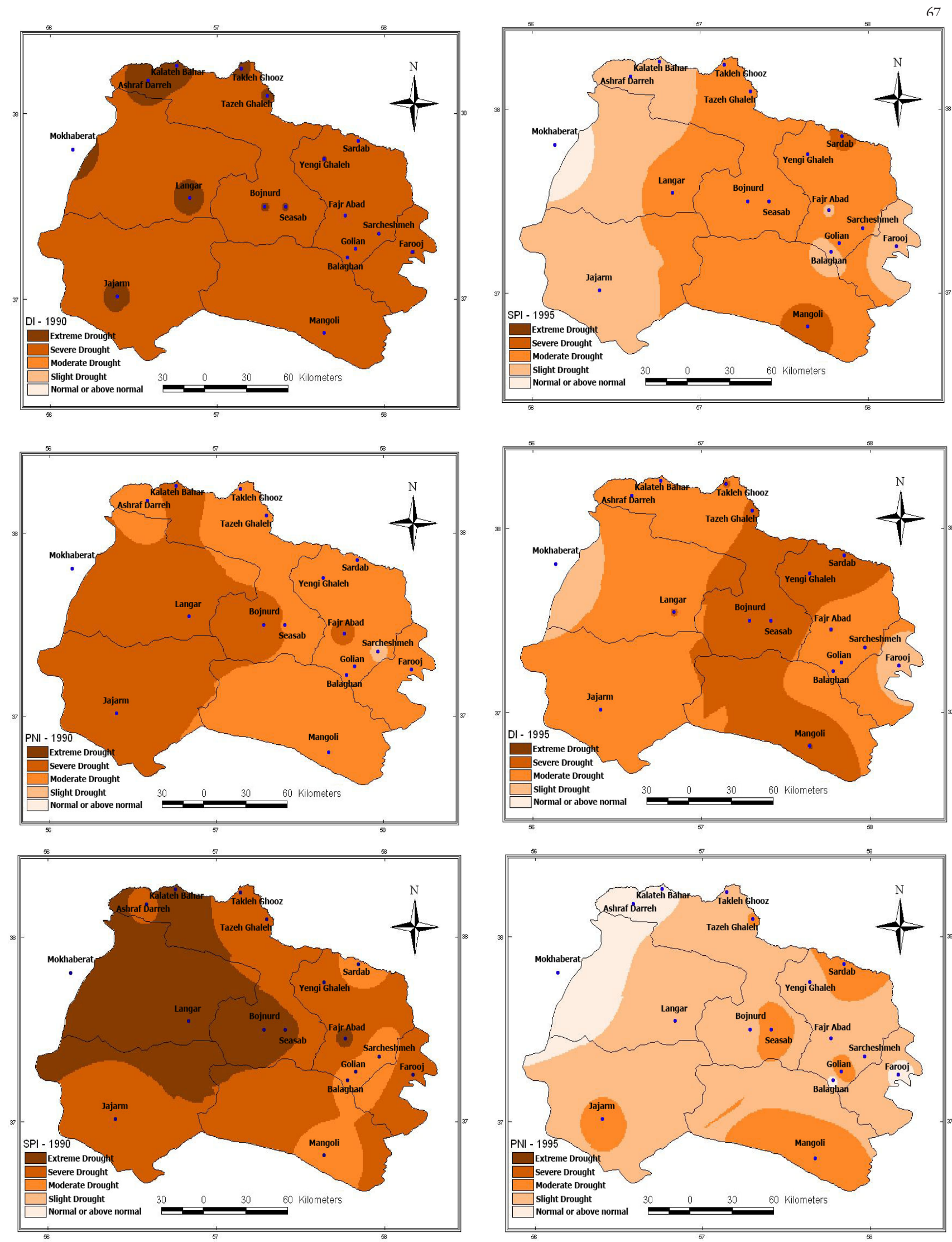

Fig. 3. Drought zone scheme for Northern Khorasan province by SPI (Standard Rainfall Index), DI (Decades Index) and PNI (Percent of Normal) indices in 1990

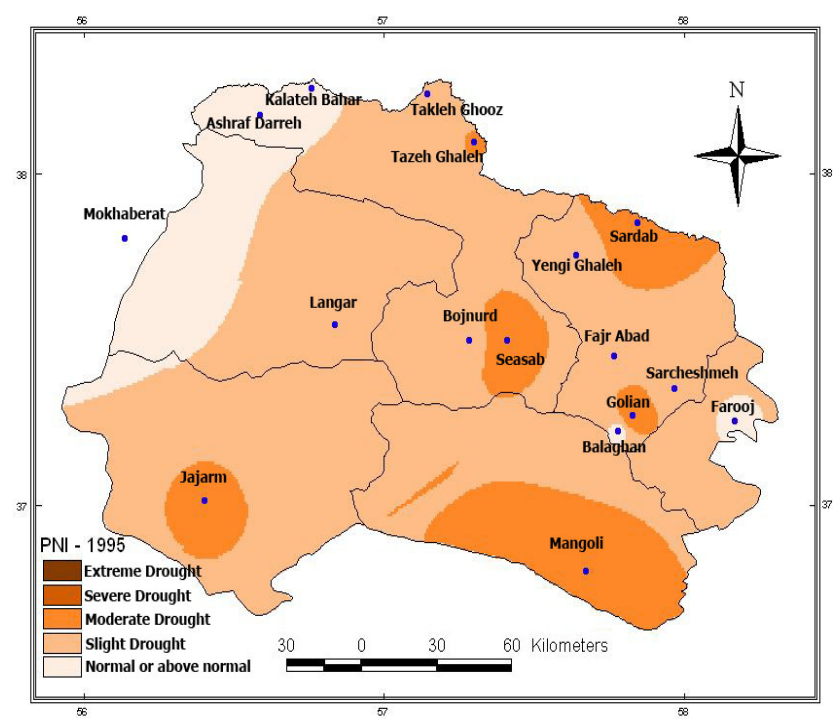

Fig. 4. Drought zone scheme for Northern Khorasan province by SPI (Standard Rainfall Index), DI (Decades Index) and PNI (Percent of Normal) indices in 1995 
68
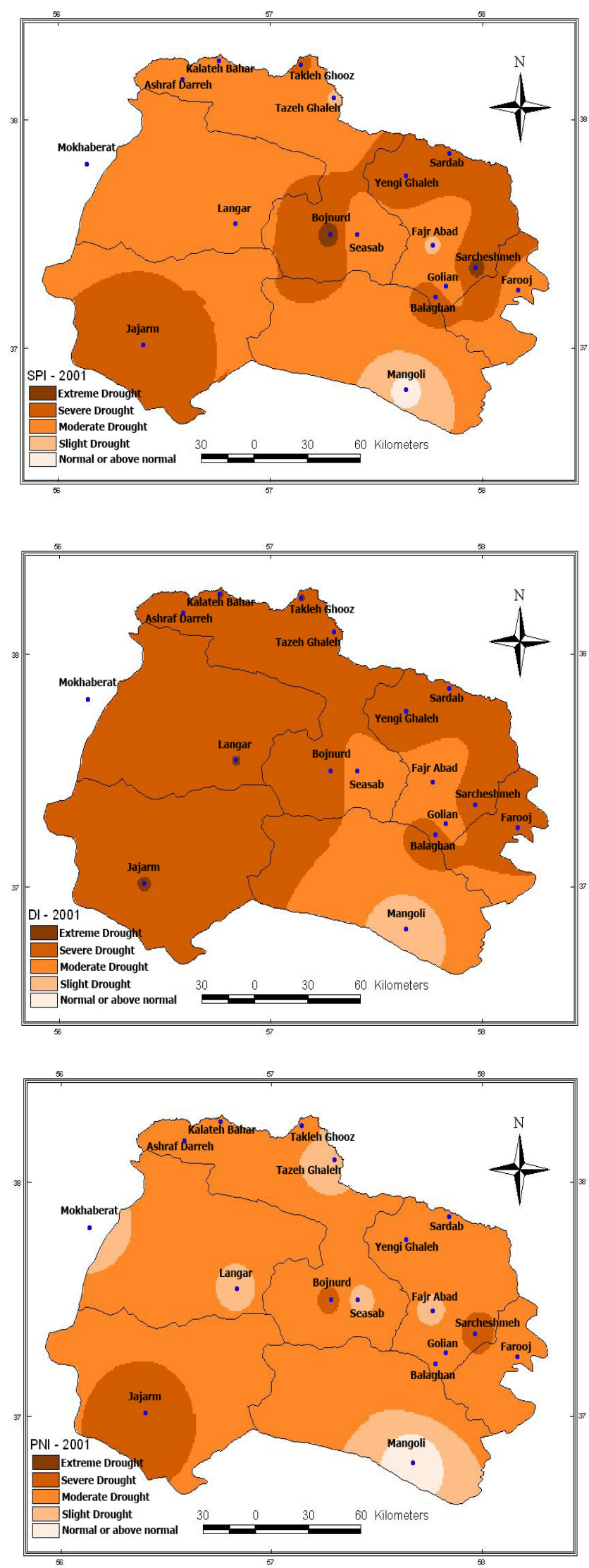

Fig. 5. Drought zone scheme for Northern Khorasan province by SPI (Standard Rainfall Index), DI (Decades Index) and PNI (Percent of Normal) indices in 2001 evaluated by various drought indices. Decades Index indicated drought situation 69 and 85 percent more severe than SPI and PNI respectively. In addition, SPI has shown severe drought intensity 55 percent more than PNI. In 30 percent of locations, DI had same drought situations with SPI, in 40 percent of stations; SPI showed equal drought situations with PNI and finally PNI showed identical conditions with DI in 14 percent of locations during drought occurrence period. In general, Decades Index demonstrated higher sensitivity to drought situation compared to other indices.

\section{Drought zone scheme}

Drought observation systems are necessary for projects deal with drought. In order to achieve this, drought indices used to quantify the drought occurrence and intensity. Generally, calculations of these indices are as point, but in order to study about extent of drought across a region showing them by map form is required. Results of this study indicated widest and most severe drought occurrence were respectively in 1990, 2001, 1995, 1989, 2000 and 1996 years during study period based on SPI, DI and PNI testing methods. Severe drought occurrence in west, northwest, and central part of province was in 2001, northeast, southwest, central part of province suffered extreme drought in 1995, and finally, severe drought occurred in southeast, northwest and central part of province was in 1990. Drought situations shown in Fig. 3 for 1990, Fig. 4 for 1995 and Fig. 5 for 2001 years, that calculated by SPI, DI and PNI testing methods. Despite the existing differences between drought indices they showed the same general trend however, indicated different intensities.

\section{Conclusions}

Drought is the world's most costly natural catastrophe, causing an average $\$ 6-\$ 8$ billion in global damages annually and affecting more people than any other form of natural disaster calamity. Given the consequences and intensity of drought, it is important to assess drought severity, but precise quantification of drought is a difficult geophysical endeavor. Northern Khorasan province of Iran situated in semi-dry region. This region had 6 years with extreme drought during study period (19 years). Central part of this province exposed with maximum drought occurrences intensity than other parts and east part of this region with minimum drought during study period. Calculation of drought by Standard Rainfall Index (SPI), Decades Index (DI) and Percent of Normal (PNI) indices showed a little deference in drought occurrence between these methods owing to the fact that nearly all methods showed same years as dry year however, drought intensities showed obvious differences between these methods. 


\section{References}

Abtew W, Obeysekera J, Shih G (1993). Spatial analysis for monthly rainfall in south Florida. Wat Res Bull 29:179188.

Akhtari R, Mahdian MH, Morid S (2007). Assessment of spatial of SPI and EDI drought indices in Tehran Province, Iran. Wat Resour Res 3:27-38.

Ansari H (2008). Drought zone scheme by standard precipitation index for Khorasan region. Geo Res 60:97-108.

Breslin P, Frunzi N, Napoleon E, Ormsby A (1999). Getting to know Arcview GIS. Enviro Sys Res Ins Pub, p. 122.

Gibbs WJ, Maher JV (1967). Rainfall deciles as drought indicators. Bureau of Meteorology Bulletin, No. 48. Comm of Aus Melb, p. 37.

Gourabi BR (2004). The study of drought using a normal percent precipitation index in central area of Guilan. Des J 2(10):249-256.

Goovaerts P (2000). Geostatistical approaches for incorporating elevation into the spatial interpolation of rainfall. J Hydrol 228:113-129.

Guttman NB (1999). Accepting the Standardized Precipitation Index: a calculation algorithm. J Am Wat Resour Assoc 35(2):311-322.
Kao SC, Govindaraju RS (2010). A copula-based joint deficit index for droughts. J Hydrol 380:121-134.

Leech N, Barrett KC, Morgan GA (2005). SPSS for intermediate Statistics. Lawr Erlb Asso Inc, Pub, p. 74.

McKee TB, Doesken NJ, Kleist J (1993). Drought monitoring with multiple time scales. In Proc, 9th Conf. on Applied Climatology, January 15-20, American Meteoro Soc Massa, p. 233-236.

Rahemi YG (2005). A study of drought and wet year assessment models for stations in East Azerbaijan Province. Iran J Natural Resour 58:517-530.

Rossi G, Vega T, Bonaccoso B (2007) Methods and tools for drought analysis and management. Sprin Pub, p. 47.

Wilhite DA (2005). Drought and Water Crises Science, Technology, and Management Issues. Taylor and Francis Ed.

Willeke G, Hosking JRM, Wallis JR, Guttman NB (1994). The national drought atlas. Ins for Wat Resour Rep 94, NDS. U.S., Army Corp of Engineers. 